\title{
LAS RELACIONES UNIÓN EUROPEA - AMÉRICA CENTRAL
}

\author{
José Ángel Sotillo*
}

La intensificación en los últimos años de las relaciones entre la Unión Europea y algunas regiones de América Latina parece haber dinamizado la vinculación eurolatinoamericana. Sin embargo, América Central, que en los ochenta se configuró como la región de América Latina donde la Europa comunitaria había mostrado una gran iniciativa, hoy parece haber quedado en un lugar marginal. Aunque se ha mantenido el diálogo con dicha región, lo cierto es que parece haber descendido algunos lugares en el interés y las prioridades de la Unión Europea. Con el nuevo panorama internacional, aquí también la geoeconomía sustituye a la geopolítica.

La década de los ochenta colocó en lo más alto el listón de las relaciones entre la Comunidad Europea y América Central. La situación conflictiva que vivía el Istmo le hacía ser centro de atención mundial, un lugar donde se jugaban las bazas de la Segunda Guerra Fría, pero en cuyo tablero aparecía por primera vez una presencia europea que actuaba conjuntamente en la Comunidad.

Se puso en marcha el llamado enfoque europeo -como alternativo al de la Administración de Estados Unidos- que vinculaba la paz en la región a la consecución simultánea no sólo del fin de los conflictos armados, sino también de la democracia y el desarrollo. La iniciativa se ponía en marcha en consonancia con la que los propios países centroamericanos desarrollaban y en apoyo explícito de los procesos de Contadora y Esquipulas, y sin que la presencia de los sandinistas en el Gobierno de Nicaragua cortara la relación con ese país. El apoyo europeo, además, se contemplaba fundamentalmente desde una dimensión regional, entendiendo que junto a problemas nacionales que deberían ser resueltos por vías nacionales, había problemas regionales que debían abordarse desde una dimensión regional.

A esas circunstancias, habría que añadir otras dos. En primer lugar, la Comunidad Europea vivía momentos de reforma y ampliación, que conducirían al Acta Única Europea (1986) y a la entrada de Portugal y de España, que dinamizarían si cabe aún más la presencia europea en la región, ya que de hecho -sobre todo España- lo venía haciendo desde una política bilateral. El Acta Única Europea formalizaba la primera puesta en escena internacional de la Comunidad Europea, la Cooperación Política
Europea, el prototipo de la futura Política Exterior y de Seguridad Común. En segundo lugar, a Europa no le interesaba que el conflicto centroamericano -aunque muy lejano en la distancia- se prolongara sin solución, dado que esa tensión podría extenderse a otros lugares y afectar, quizá, al propio continente europeo, que ya comenzaba a vivir las primeras convulsiones que desembocarían en los acontecimientos de 1989. Tal como se recoge en un informe de IRELA ', "Los países de la UE, durante la Guerra Fría, comenzaron a preocuparse por los peligros que entrañaban para el proceso de distensión internacional las crisis regionales y los conflictos armados localizados, así como por las posibles repercusiones sobre sus esfuerzos para mantener la paz y establecer formas de cooperación entre los Estados del Este y del Oeste del continente europeo. Es por ello que el entonces Ministro de Asuntos Exteriores de España, Fernando Morán, expresó su preocupación de que Europa se viese afectada por el despliegue militar efectuado por EE.UU. en América Central y por una posible intervención militar norteamericana en la región".

Así pues, solidaridad e interés se mezclaron para dar vida a una gran actividad diplomática, política y solidaria con América Central, mucho más importante si tenemos en cuenta que se realizaba de forma manifiestamente contraria a la que defendía Estados Unidos bajo la presidencia de Reagan.

La forma en que la Comunidad materializó esa relación supuso una innovación en los mecanismos de acción exterior comunitarios, aunque habría que decir con exactitud de los Estados miembros, puesto que fueron éstos quienes siempre tuvieron la competencia sobre estos asuntos, aunque la vía elegida para llevarla a cabo fuera por medio de la Comunidad. El 28 de septiembre de 1984 se inauguraba en San José la Primera conferencia Ministerial sobre el diálogo político y la cooperación económica entre América Central y la Comunidad Europea, compuesta entonces por diez Estados miembros, aunque España formaba parte

1. Irela: Diez años del Proceso de San José. Un balance de la cooperación Unión Europea - América Central, Madrid, 1994, pp. 12-13. Ver también Morán, Fernando: "El papel de Europa en Centroamérica”, Tiempo de Paz, n. 7, otoño 1985. 
también de la delegación europea. Los conflictos internos, aunque con una clara dimensión regional, la crisis económica y financiera, y la falta de sistemas democráticos en buena parte de los países centroamericanos fueron los ejes de la acción comunitaria, que se convertiría en el primer donante de Ayuda Oficial al Desarrollo de la región.

Esa proyección en América Central ha llevado al Parlamento Europeo a considerar que "los logros alcanzados en el marco del diálogo ministerial de San José entre la Unión Europea y Centroamérica iniciado en septiembre de 1984 constituyen uno de los mayores éxitos cosechados hasta el momento por la política exterior común de la Unión Europea" ${ }^{2}$.

A pesar de ese balance positivo, se han señalado algunos aspectos que revelan los condicionantes y el alcance de esa relación. Para Gomes Saraiva ${ }^{3}$ " "Sin embargo, a pesar de la mayor importancia otorgada por la Comunidad a la región y de la disposición para aumentar la ayuda económica, quedaron claras algunas limitaciones. Primero dejó entrever que un acuerdo se orientaría más hacia el campo de la cooperación para el desarrollo, sobre todo para la integración regional, como una de las líneas maestras de la actuación europea hacia América Latina durante el período. Segundo, en el campo comercial no se podía esperar mucho de la Comunidad, en función de sus otras prioridades de comercio exterior y de las restricciones relativas a su política de integración".

Junto a ese apoyo político y solidario, no conviene olvidar que se mantiene una tensión económica entre ambas regiones, por lo que podemos afirmar, con Sanahuja, que ha existido un encuentro político y un desencuentro económico. En el ámbito económico "la respuesta europea ha sido contradictoria y desalentadora, y a largo plazo ha debilitado la credibilidad y la eficacia del diálogo político... la Comunidad ha apoyado la liberalización económica y la diversificación de las exportaciones centroamericanas hacia productos no tradicionales, pero ha mantenido en lo esencial su proteccionismo... En suma, la Comunidad sólo ofreció el tradicional modelo de cooperación de 'ayuda sin comercio' (aid, not trade) frente al 'comercio y ayuda' (traid and aid) concecido a los países ACP, o el 'comercio, no ayuda' (trade, not aid) tradicionalmente demandado por los países en desarrollo" ${ }^{4}$.

La relación regional se formalizó por medio de un Acuerdo de Cooperación, adoptado con motivo de la segunda conferencia, celebrada en Luxemburgo los días 11 y 12 de noviembre de 1985; el Acuerdo entró en vigor el 1 de marzo de 1987. El Acuerdo, de los denominados de segunda generación, incluía como objetivos la profundización de la cooperación económica, el fortalecimiento e institucionalización de las relaciones mutuas, el impulso al proceso de integración económica centroamericana y el incremento de la asistencia financiera y técnica y de la cooperación científica.

El Acuerdo de 1985 fue sustituido por el Acuerdo Marco de Cooperación, firmado el 27 de febrero de 1993, en el que se incluye, en el artículo 1 el fundamento democrático de la cooperación ("Las relaciones de cooperación entre la Comunidad y Centroamérica, y todas las disposi- ciones del presente Acuerdo se basan en el respeto de los principios democráticos y de los derechos humanos, que inspiran las políticas internas e internacionales tanto de la Comunidad como de Centroamérica, y que constituyen un elemento fundamental del Acuerdo"). Quizá las pretensiones del Acuerdo hayan quedado limitadas por el mayor acercamiento europeo a otras regiones y países latinoamericanos, con el objetivo de establecer zonas de libre comercio, como el caso del Acuerdo con MERCOSUR.

A medida que se han celebrado las conferencias ministeriales -con carácter anual excepto en 1986-, podemos constatar un descenso paulatino en la intensidad del diálogo. Se han ido cumpliendo los grandes objetivos fijados en 1984, como los procesos de pacificación y democratización, lo que ha dado lugar a una cierta despolitización de las relaciones en favor de una tecnificación, en cuanto a las acciones a poner en marcha para consolidar los sistemas democráticos.

La pérdida de intensidad produjo cambios en los mecanismos que institucionalizan el diálogo. En la XII Conferencia Ministerial (Florencia, 21 de marzo de 1996), se decidió la renovación del diálogo, dándole nuevo formato: se celebrarían reuniones a nivel ministerial cada dos años, reuniones con la 'troika' en los otros años, reforzándose el papel de la Comisión Mixta. Las prioridades que si fijan para la cooperación son: la consolidación del Estado de derecho, la reforma del Estado, políticas sociales, integración regional e inserción en la economía mundial. Es significativo que al año siguiente, en la reunión de La Haya, la primera según el nuevo formato fijado en Florencia, de la 'troika' sólo acudiera uno de los ministros europeos de Asuntos Exteriores.

La XIV Conferencia se celebró en la capital de Costa Rica, los días 10 y 11 de febrero de 1998. Considerada como muestra de la consolidación del nuevo diálogo entre ambas regiones, en la reunión se abordaron las siguientes cuestiones: el proceso de integración centroamericano (sobre todo teniendo en cuenta la reunión de presidentes que tuvo lugar en septiembre de 1997 en Managua); la extensión a los productos industriales centroamericanos del régimen especial del Sistema de Preferencias Generalizadas; las relaciones económicas y comerciales; la cuestión de los plátanos; y la futura cumbre Unión Europea - América Latina. Además se trataron otras cuestiones, como la cooperación en materia de consolidación del Estado de derecho y de seguridad de los ciudadanos, los derechos humanos y el respeto de los derechos fundamentales de los trabajadores, la lucha contra el tráfico de drogas y el crimen organizado, y la lucha contra las minas antipersonas. Con el aval que supone el constante apoyo a la integración

2. Resolución sobre Comunicación de la Comisión al Consejo rela tiva a la renovación del diálogo de San José entre la Unión Europea y Centroamérica, adopta el 16 de enero de 1987. DOCE C 33, 3.2.97, p. 91 .

3. Gomes Saraiva, Miriam: Politica externa europea. El caso de los diálogos grupo a grupo con América Latina de 1984 a 1992. Buenos Aires, GEL, 1996, p. 170.

4. Sanahuja, José Antonio: Renovando el Diálogo de San José. El futuro de las relaciones entre América Central y la Unión Europea, Londres, Instituto Católico de Relaciones Internacionales, 1996, p. 4. 
centroamericana y ser el principal donante de ayuda al desarrollo -el $62 \%$ del total- el vicepresidente de la Comisión Europea, Manuel Marín, pasó revista al estado actual de ese proceso y destacó las líneas fundamentales de la acción europea. Si hace veinte años el objetivo fundamental era poner fin a la guerra, "ahora el principal condicionante para la concesión de ayudas es la gobernabilidad el país. Justicia, policía, políticas fiscales y aduaneras, parlamentarismo... Marín insiste constantemente en que Europa tiene sus propias exigencias, que le importa poco el color político de los Gobiernos mientras provengan de un proceso democrático y pide la regionalización para no quedar solos ante la apisonadora de la economía global" s.

En la XIV Conferencia reaparecen el encuentro político y el desencuentro económico. Junto al reconocimiento, por un lado, de los esfuerzos centroamericanos en favor de la democratización y el desarrollo y, por otro, del apoyo europeo a esas iniciativas, algunos puntos del Comunicado conjunto desvelan las dificultades que permanecen constantes: "Las Partes se felicitaron por el impacto positivo del régimen de las preferencias generalizadas especiales para los productos agrícolas, otorgado a Centroamérica por la Unión Europea desde el 1 de enero de 1997, el cual ha contribuido a una mayor integración de Centroamérica en la económica mundial, ampliando sus exportaciones e importaciones en proporción a su producción, lográndose una mejor posición en algunos sectores de la producción agrícola no tradicional y mejorando las condiciones de vida de amplios grupos sociales mediante la creación de nuevas fuentes de empleo, entre otros para las mujeres y los indígenas. Asimismo, la Parte centroamericana reiteró su petición de que se aplique un régimen para los productos industriales similar al otorgado a los países de la Comunidad Andina, a fin de consolidar el esfuerzo centroamericano para integrarse en el nuevo espacio económico global. Este generaría ventajas importantes para las empresas europeas abastecedoras de bienes de capital y tecnología destinados a la modernización de las industrias centroamericanas. La Unión Europea tomó nota de la petición centroamericana de que se adopte un régimen de preferencias especiales para los productos industriales. La Comisión Europea examinará esta cuestión a la luz de los criterios pertinentes al elaborar la propuesta de renovación del sistema de preferencias generalizadas previstas para este año". Las espadas -económicas- siguen pues en alto.

Pasando revista a las relaciones entre ambas regiones, Stevens ${ }^{6}$ ha puesto de relieve el interés que América Central tiene en su diálogo político con la Unión Europea: “ ¿No es cierto que ésta otorga el 58\% de toda la ayuda exterior que recibe el Istmo, absorbe el 19\% de toda su exportación y se mantiene desde hace años como el primer comprador de bananas y café proveniente de esta región? El Istmo considera sus relaciones con la UE como alternativa útil (quizá se pueda incluso recurrir al término de instrumento de presión) para disminuir su alta dependencia respecto de EE.UU. o con vistas a ampliar su margen de negociaciones en Washington. Por lo demás, la UE ha demostrado ser su socio más cooperativo cuando se trata de defender la democracia, los derechos humanos y la integración regional”. El diálogo sólo recuperará su atractivo cuando la agenda aborde cuestiones que tengan un valor geopolítico común y específico (el problema de las drogas, la cuestión del medio ambiente, el acceso al mercado y la cooperación económica y política en la región) o implique importantes 'valores blandos' (la democracia, los derechos humanos, el desarme civil, el problema de las minas antipersonas, el tráfico internacional de armas). Stevens señala algunas de las carencias actuales del proceso de San José: "Los Estados miembros de la UE como los países de América Central tienden a reprocharse mutuamente no preparar suficientemente los debates, adoptar una posición demasiado teórica, no desviarse de los discursos preparados para confrontarse en un diálogo abierto y espontáneo y no presentar propuestas concretas de acción”.

A diferencia de la década de los ochenta, en la actualidad a la Unión Europea ya no le intresa tanto América Central, ni América Central necesita tanto a la Unión Europea. Parece también que, como le sucede a muchas parejas, más de diez años de relaciones acaban por traer la rutina y diluir progresivamente los lazos que antes les mantenían más unidos. La normalización de las relaciones ofrece la imagen del cumplimiento de los objetivos fijados, pero también da la impresión de que la Unión Europea ha dejado a América Central en un segundo lugar, fijando sus prioridades en las economías emergentes.

Un factor fundamental para entender el futuro de las relaciones entre la Unión Europea y América Central, son las profundas transformaciones que ambas regiones están viviendo actualmente y que, quizá, les hace concentrar sus esfuerzos más hacia adentro que hacia afuera. El Tratado de Amsterdam, sucesor del Tratado de Maastricht, no acaba de definir el futuro de la Unión Europea, por lo que quedan pendientes cuestiones tan importantes como la financiación, la ampliación, la reforma institucional, los fondos estructurales, la reforma de las principales políticas... y, además, hacia fuera tiene que definir el nuevo Convenio de Lomé (al que se ha invitado a asociarse a Cuba, que ha aceptado; invitación que podría extender a algún país centroamericano), es decir, el principal mecanismo de relaciones entre la Comunidad y los países subdesarrollados, la relación con los países del Mediterráneo y, por último, con los países de Asia y de América Latina. Con América Latina algunas de esas dudas puede que se despejen en la reunión que los jefes de Estado y de Gobierno de la Unión Europea y de América Latina celebrarán en Rio de Janeiro en el primer trimestre de 1999, aunque está por ver si las expectativas que despierta no quedarán lejos de los resultados que se consigan.

También América Central, cuyo proceso de integración es unos de los más antiguos, vive momentos de revitalización. En la cumbre de Presidentes celebrada en Managua, el 2 de septiembre de 1997, los máximos representantes se mostraron "conscientes de que este esfuerzo

5. Ver El Pais (Internacional), de 11 de febrero de 1998.

6. Stevens, Willy J.: “El proceso de San José”, Politica Exterior, n. 61, enero/febrero 1998, pp. 143-157. 
requiere de un inequívoco compromiso con el ideal centroamericanista"; bajo ese espíritu se acordó "iniciar el proceso de constitución, gradual y progresivo, de la Unión Centroamericana".

A la vista de esa situación, la acción de la Unión Europea dependerá del grado de avance de la integración centroamericana. Con motivo de la XIV Conferencia Ministerial, el Comisario Manuel Marin resaltaba esa circunstancia: "¿Puede sobrevivir una América Central dividida en pequeñas economías, con pequeñas poblaciones, con un sistema económico compartimentado...? Es difícil, muy difícil, por no decir imposible". La Comisión Europea garantiza que proseguirá y enriquecerá su ayuda, pero Centroamérica es la que debe encontrar el mecanismo para pasar de las pequeñas economías a una economía capaz de desempeñar una función en las negociaciones de la zona de librecambio de las Américas (que deberían emprenderse por los 34 países del continente americano en la conferencia de Santiago de chile, el próximo mes de abril) y en las negociaciones con la Unión Europea, señaló el vicepresidente de la Comisión. Parece que "Centroamérica no ha comprendido" que la integración es necesaria frente al cambio político y económico del próximo siglo ${ }^{7}$.
Como hemos manifestado en otra ocasión, "En 1984 la Comunidad Europea y América Central adoptaron un compromiso mutuo en torno a la paz, la democracia y el desarrollo. Trece años después, a pesar de los innegables avances registrados, estos temas están aún vigentes. La Unión Europea debe seguir concediendo una atención especial a Centroamérica, adaptando el contenido del diálogo y sus métodos de acción a las nuevas realidades de la región y a la Política Exterior y de Seguridad Común (PESC) prevista en el Tratado de Maastricht. La propia Comisión ha señalado que la Unión Europea tiene un compromiso moral con la región, y que no puede dejarla en el olvido cuando la democracia y la paz están en proceso de consolidación" ${ }^{8}$.

7. Boletín Europe, n. 4120, de 12 de febrero de 1998.

8. El Parlamento Europeo y el proceso de integración centroamerica na, Informe elaborado por José Antonio Sanahuja y José Ángel Sotillo. Parlamento Europeo, Luxemburgo, 1997, p. 96.

\title{
RESUMEN
}

En este artículo se presenta la evolución reciente de las relaciones entre la Unión Europea y América Central. $\mathrm{El}$ autor señala las razones por las cuales se pasó de una etapa de intensa relación entre ambas regiones, en los años ochenta, a una de relativo enfriamiento en los noventa. En general se pone de manifiesto una tendencia al acuerdo político y al desacuerdo en materia económica.

Palabras clave: América Central, Unión Europea, relaciones internacionales, integración.

\begin{abstract}
The article analyses the recent evolution of European Union and Central America relationship. The author defines the main reasons of the change from an intense relationship between boht regions during the eihties, to a certain weakening of the links. We can find an evident trend of consensus in political matters coexisting with a dynamic of disagreement on economical questions.

Key words: Central America, European Union, international relations, integration.
\end{abstract}

